\title{
Low polarised emission from the core of coronal mass ejections
}

\author{
M. Mierla ${ }^{1,2,3}$, I. Chifu ${ }^{4,5}$, B. Inhester ${ }^{4}$, L. Rodriguez ${ }^{1}$, and A. Zhukov ${ }^{1,6}$ \\ 1 Solar-Terrestrial Center of Excellence - SIDC, Royal Observatory of Belgium, Brussels, Belgium \\ 2 Institute of Geodynamics of the Romanian Academy, 020032 Bucharest, Romania \\ e-mail: marilena@geodin.ro \\ 3 Research Center for Atomic Physics and Astrophysics, Faculty of Physics, University of Bucharest, Romania \\ ${ }^{4}$ Max-Planck-Institut für Sonnensystemforschung, Katlenburg-Lindau, Germany \\ 5 Astronomical Institute of the Romanian Academy, Bucharest, Romania \\ 6 Skobeltsyn Institute of Nuclear Physics, Moscow State University, Russia
}

Received 10 December 2010 / Accepted 11 April 2011

\section{ABSTRACT}

\begin{abstract}
Aims. In white-light coronagraph images, cool prominence material is sometimes observed as bright patches in the core of coronal mass ejections (CMEs). If, as generally assumed, this emission is caused by Thomson-scattered light from the solar surface, it should be strongly polarised tangentially to the solar limb. However, the observations of a CME made with the SECCHI/STEREO coronagraphs on 31 August 2007 show that the emission from these bright core patches is exceptionally low polarised.

Methods. We used the polarisation ratio method of Moran \& Davila (2004) to localise the barycentre of the CME cloud. By analysing the data from both STEREO spacecraft we could resolve the plane-of-the-sky ambiguity this method usually suffers from. Stereoscopic triangulation was used to independently localise the low-polarisation patch relative to the cloud.

Results. We demonstrated for the first time that the bright core material is located close to the centre of the CME cloud. We show that the major part of the CME core emission, more than $85 \%$ in our case, is $\mathrm{H} \alpha$ radiation and only a small fraction is Thomson-scattered light. Recent calculations also imply that the plasma density in the patch is $8 \times 10^{8} \mathrm{~cm}^{-3}$ or more compared to $2.6 \times 10^{6} \mathrm{~cm}^{-3}$ for the Thomson-scattering CME environment surrounding the core material.
\end{abstract}

Key words. Sun: coronal mass ejections (CMEs) - Sun: filaments, prominences - polarization - techniques: polarimetric

\section{Introduction}

The core of coronal mass ejection (CME) clouds occasionally exposes very bright concentrated patches in white-light coronagraphs. They are interpreted as cool plasma material from a prominence that was embedded inside the streamer environment of the CME before the eruption. During the eruption process, the prominence is then expelled along with the surrounding streamer plasma.

Poland \& Munro (1976) report one such observation made on 21 August 1973, 15:11 UT with the Skylab white-light coronagraph and its HeII $30.4 \mathrm{~nm}$ spectroheliograph. About $18 \mathrm{~min}$ before, an $\mathrm{H} \alpha$ image taken at the Sacramento Peak Observatory had shown bright patches extending out to $1.42 R_{\odot}$ but fading in intensity with time. Even though the coronagraph field-of-view was limited to above $1.5 R_{\odot}$, it was concluded that $\mathrm{H} \alpha$ radiation contributed to the white-light image because its signal was less polarised in some bright patches than in the surrounding region.

$\mathrm{H} \alpha$ radiation is the result of the electronic $j=3 \rightarrow j=2$ transition of the hydrogen atom. In equilibrium at an electron temperature below $50000 \mathrm{~K}$, the $j=3$ level is populated much more by absorption of the ambient $\operatorname{Ly} \beta$ radiation than by absorption of photospheric $\mathrm{H} \alpha$. This causes a substantial decrease in polarisation of the emitted $\mathrm{H} \alpha$ radiation below the theoretical maximum value of $30 \%$ for pure resonant scattering (Poland \& Munro 1976). Besides, the Hanle effect caused by the coronal magnetic field (Sahal-Brechot et al. 1977; Heinzel et al. 1996) and collisional depolarisation (Bommier et al. 1986) reduce the amount of polarisation even further. As a result, the linear polarisation of $\mathrm{H} \alpha$ radiation observed in prominences well above the limb ranges from a fraction of a percent (Gandorfer 2000; Wiehr $\&$ Bianda 2003) to a few percent (Leroy et al. 1984).

The white-light emission of the solar K-corona originates in Thomson-scattering of photospheric light by free electrons. Detailed description of the Thomson-scattering theory can be found in various papers (e.g., Minnaert 1930; van de Hulst 1950; Billings 1966). The anisotropy of the incident light causes the observed scattered radiation to exhibit a polarisation parallel to the visible limb. The degree of polarisation depends on the distance from the solar surface and on the scattering angle to the observer. Indeed, it has been proposed to use the observed degree of polarisation to estimate the distance of the coronal scattering volume off the plane of the sky (POS, e.g., Moran \& Davila 2004; Dere et al. 2005; Vourlidas \& Howard 2006). Hence, a reduction of polarisation of a white-light signal from the corona can in principle also be explained by a geometric effect, and Poland and Munro's conclusion only holds if it is assumed that the $\mathrm{H} \alpha$ material is well embedded in the CME cloud of enhanced electron density and is located close to the POS of the observer.

In this Letter, we report the observation of a similar incidence with the COR1 coronagraph on board the two STEREO spacecraft $\mathrm{A}$ and $\mathrm{B}$. In addition to the polarisation measurements from which we determine the azimuthal barycentre position of the CME plasma, a stereoscopic triangulation of the lowpolarisation patch proves that the prominence material is well embedded inside the CME. 


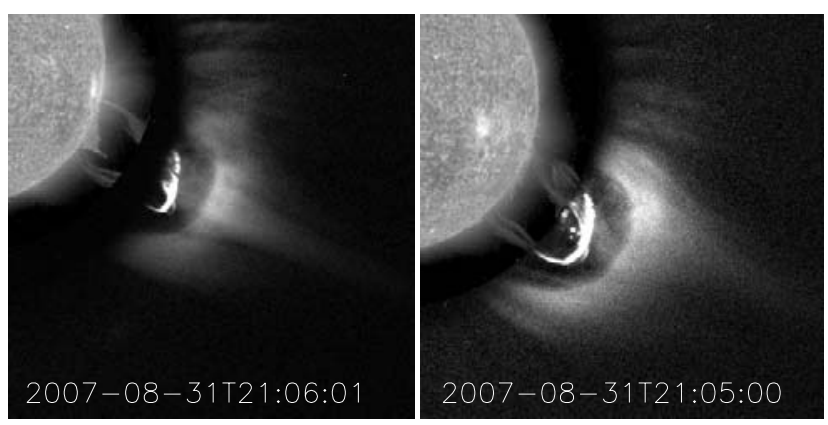

Fig. 1. Combined images of EUVI and COR1 on board STEREO A and B. Both A and B images are synchronous with respect to the time on the Sun.

\section{Observations}

EUVI and COR1 are the multi-wavelength EUV telescope and the innermost coronagraph of the Sun Earth Connection Coronal and Heliospheric Investigation (SECCHI) instrument suite (Howard et al. 2008) aboard the twin Solar TErrestrial RElations Observatory spacecraft (STEREO, see Kaiser et al. 2008). Each of the COR1/STEREO telescopes has a field of view from 1.4 to $4 R_{\odot}$ and observes in a white-light waveband $22.5 \mathrm{~nm}$ wide centred at the $\mathrm{H} \alpha$ line at $656 \mathrm{~nm}$ (Thompson \& Reginald 2008). The COR 1 coronagraphs take polarised images at three different polarisation angles at 0,120 , and 240 degrees. These primary data allow to derive Stokes $I, U$ and $Q$ components and finally total $(t B)$, polarised $(p B)$ and unpolarised brightness $(u B=t B-p B)$ images.

The start of a prominence eruption was observed in EUVI HeII bandpass images at $30.4 \mathrm{~nm}$ on 31 August 2007, at around 19:00 UT. Approximately eight hours before, a dark filament was detected about $100000 \mathrm{~km}$ south of an active region close to the west limb in the $\mathrm{H} \alpha$ images of Kanzelhöhe Observatory, which had disappeared the following day (http://cesar. kso.ac.at/halpha2k/archive/2007). At about 21:00 UT, the HeII prominence had risen to $1.5 R_{\odot}$ and appeared co-spatial with the bright core of a structured CME detected in COR1 images (Fig. 1, see for e.g. Cremades \& Bothmer 2004, for the definition of a structured CME).

Preliminary COR1 images of the event studied here revealed patches of extremely low polarisation in the bright CME core. These patches of low polarisation could faintly be detected even out to about $7 R_{\odot}$ in COR2 at about 2:00 UT the next day. COR2 is the outer coronagraph of STEREO/SECCHI, which covers distances from 3 to $15 R_{\odot}$. The outward velocity of these patches started from about $170 \mathrm{~km} \mathrm{~s}^{-1}$ in COR1 and accelerated to $240 \mathrm{~km} \mathrm{~s}^{-1}$ in the COR2 field of view.

\section{Data analysis}

For a more quantitative analysis we removed the background contribution by subtracting a minimum intensity $p B$ and $t B$ image from each $p B$ and $t B$ images, respectively. The minimum images were obtained over a time range of 12 hours, centred at the launch time of the CME. As a result, we obtain the brightness of the CME alone. In Fig. 2 we display the resulting ratio $p B / u B$ of polarised to unpolarised image intensities. From both instruments we see bright patches of extremely low polarisation (red, $p B / t B \approx 0.1$ ) at about $1.5 R_{\odot}$ inside the strongly polarised CME cloud (grey, $p B / t B \approx 0.5$ ).

The degree of polarisation of Thomson-scattered light by coronal electrons is a function of the scattering angle between

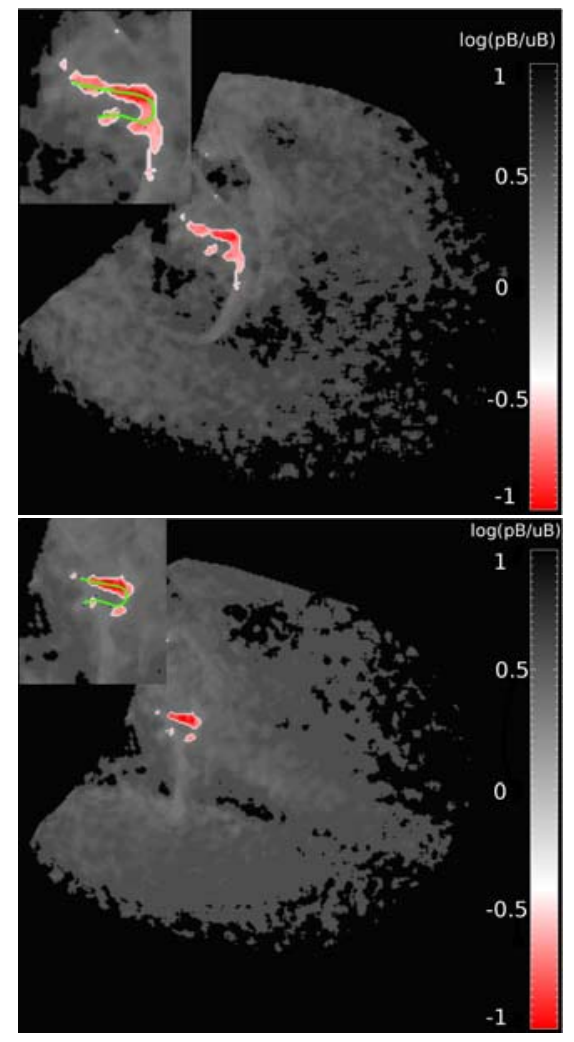

Fig. 2. Ratio $p B / u B$ of polarised to unpolarised light from COR1 of STEREO spacecraft A (upper panel) and B (lower panel). The ratio is colour-coded and the red patches mark low polarised values. The upper left insert is a zoom of this region with the green line representing the projection of the $3 \mathrm{D}$ curve fit to the patches location obtained from stereoscopic triangulation.

the incident light direction and the direction towards the observer (Billings 1966). A scattering location close to the POS has a 90 degree scattering angle and yields a high polarisation parallel to the limb. A scattering location far off the POS corresponds to nearly forward/backward scattering, which is hardly polarised. This effect allows us to estimate an effective scattering angle from the observed degree of polarisation for each pixel that can be related to an effective distance of the scattering location from the POS. Moran \& Davila (2004) and Dere et al. (2005) introduced this method, called polarisation ratio method (PR), to construct the azimuthal barycentre plane of a CME. We used this technique to infer the propagation direction of the CME discussed here (Mierla et al. 2009). The method is applied here to polarisation data from COR1/STEREO A and B taken at around 21:30 UT. At each pixel of the images, the ratio $p B / u B$ is calculated and converted into the effective scattering distance along the line-of-sight from the POS. Owing to the forward/backward symmetry of Thomson scattering, the brightness ratio does not indicate whether the scatterer is in front of or behind the POS. This ambiguity can be resolved, however, by matching the scattering locations derived from STEREO A and B observations.

In Fig. 3 we display the result of this analysis as viewed from a direction along the northward normal of the STEREO mission plane. The green and blue dots represent the scattering location derived from COR1/STEREO A and B pixels of the CME cloud, respectively. Obviously, they match well if the barycentre of the CME is assumed in front of the POS of STEREO A but behind the POS of STEREO B. The only exception are the "horns" that extend to the far right for STEREO A and to the far 


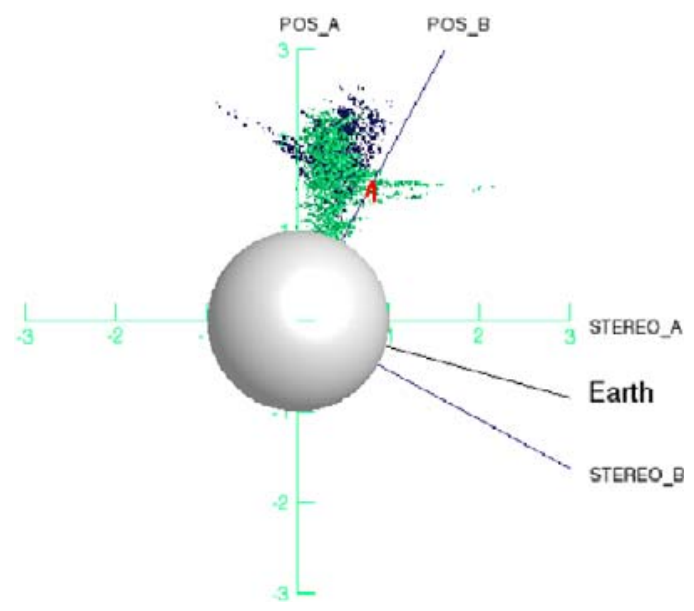

Fig. 3. Polarisation ratio reconstruction of the barycentre plane of the CME on 31 August 2007, 21:30 UT (see Sect. 3.1 for details).

left for STEREO B pixels. These "horns" result from the lowpolarisation patches observed in each of the COR1 images. If they were produced by Thomson scattering, the low-polarisation patch would have to be located at the tip of the "horns" in Fig. 3, i.e., far away from the centre of the CME. However, these positions from STEREO A and B are several solar radii apart and are therefore inconsistent.

An independent estimate of the location of the lowpolarisation patch can be obtained by stereoscopy, provided we assume that the patches we see in both coronagraphs are the same object. Both in brightness (see Fig. 1) and in polarisation ratio (see Fig. 2) the patches displayed a high contrast and a sharp boundary with respect to the CME environment. The COR1 instrument of STEREO B seems somewhat less sensitive because not all features of the STEREO A patch can be matched to an equivalent signal in the STEREO B image. Yet we could determine a loop-like 3D curve, the projections of which trace out the patches in COR1/STEREO B and the major structures in COR1/STEREO A. These projections are drawn in green into the inserts of Fig. 2.

The 3D curve is again projected into Fig. 3, in red. The triangulation of the patches locates it very close to the barycentre plane of the CME. Note that the cloud in Fig. 3 does not show the full extent of the CME, but is only an approximation of its azimuthal barycentre plane. The PR method cannot resolve the azimuthal extent of a CME (see Mierla et al. 2011).

\section{Discussion}

Low polarisation in coronagraph images can have several explanations: 1) F-corona emission (e.g., Morgan \& Habbal 2007), 2) Thomson scattering from enhanced plasma density far away from the POS (e.g., Billings 1966), and 3) $\mathrm{H} \alpha$ emission (e.g., Poland \& Munro 1976). F-corona contributions can be ruled out because it forms a diffuse background and does not vary rapidly in time. The subtraction of the minimum background intensities from our images should have removed any F-corona contribution. The interpretation of the low-polarisation patches by Thomson scattering can also be ruled out. We showed in the previous section that this assumption leads to different locations of the scatterer for STEREO A and B observations, which are both inconsistent with the results from the stereoscopic triangulation.

We are therefore left with the last explanation. $\mathrm{H} \alpha$ patches in white-light coronagraph images have only rarely been reported, even though bright amorphous structures are often seen in CME clouds. Coronal mass ejections often appear in coronagraph images as three-part structures composed of a bright leading edge, a dark cavity, and a bright core, which are associated with the compressed solar plasma ahead of the ejecta, the erupting magnetic flux rope, and the cool and dense prominence plasma, respectively (e.g., Cremades \& Bothmer 2004). However, a convincing identification of the white-light core of the CME with the cool prominence material is relatively rare. Even if recent studies have demonstrated a strong connection between prominence eruptions and CMEs, the correlation is not always one-to-one.

It is not quite clear how much of the emission from the bright core structures are produced by Thomson scatter from its enhanced plasma density and how much stems from $\mathrm{H} \alpha$ radiation. Because we observed the patches at an outward velocity above $100 \mathrm{~km} \mathrm{~s}^{-1}$, their $\mathrm{H} \alpha$ resonance is well outside of the $\mathrm{H} \alpha$ absorption line from the solar surface spectrum. Even though the depth of the absorption is $1 / 7$ of the surrounding continuum, the $\mathrm{H} \alpha$ radiation emitted from the rising patch is only little enhanced by this Doppler brightening effect because of the complicated balance of the electronic level population of hydrogen atoms exposed to the solar spectrum (e.g., Hyder \& Lites 1970).

The observed brightness $B_{\text {patch }}$ of the $\mathrm{H} \alpha$ patch in the image is in fact a line-of-sight (LOS) superposition of the radiation from the three different sources: the $\mathrm{H} \alpha$ radiation, $B_{\mathrm{H} \alpha}$, and the Thomson scatter, $B_{\mathrm{Th}}$, from inside the $\mathrm{H} \alpha$ cloud and the ambient Thomson scatter, $B_{\mathrm{Th}}$, along remaining part of the LOS through the patch. The latter contribution can roughly be assumed equal to the Thomson scatter measured close to, but outside the $\mathrm{H} \alpha$ patch.

Both the total and the polarised intensities add (assuming that the polarisation direction in all three components is the same),

$$
\begin{gathered}
t B_{\text {patch }}=t B_{\mathrm{H} \alpha}+t B_{\mathrm{Th}^{\prime}}+t B_{\mathrm{Th}} \\
p B_{\mathrm{patch}}=p B_{\mathrm{H} \alpha}+p B_{\mathrm{Th}^{\prime}}+p B_{\mathrm{Th}} .
\end{gathered}
$$

We find that the total brightness of the $\mathrm{H} \alpha$ patch (around $1-5 \times 10^{-8} \mathrm{MSB}$, where MSB is mean solar brightness) is about 10 times as high as a typical value in the Thomson-scattering area (around $\left.1-5 \times 10^{-9} \mathrm{MSB}\right)$, i.e., $t B_{\text {patch }} \simeq 10 t B_{\mathrm{Th}}$. According to the Thomson-scattering theory (Billings 1966), the calibrated value of $t B_{\mathrm{Th}}$ corresponds to an electron column density along the LOS of $1.8 \times 10^{17} \mathrm{~cm}^{-2}$. For a depth of the CME cloud of about $1 R_{\odot}$, this yields an excess density of the CME over the streamer background of $2.6 \times 10^{6} \mathrm{~cm}^{-3}$.

For the polarisation ratio we find (see Fig. 2)

$r=\frac{p B}{t B}=\frac{\frac{p B}{u B}}{1+\frac{p B}{u B}} \simeq \begin{cases}0.5 & \text { for } r_{\mathrm{Th}} \\ 0.1 & \text { for } r_{\mathrm{patch}},\end{cases}$

where $r=p B / t B$ is the polarisation ratio of the respective component. For Thomson scatter, $r_{\mathrm{Th}}$ should depend only on the distance from the solar surface, hence we can assume $r_{\mathrm{Th}} \simeq r_{\mathrm{Th}^{\prime}}$. Obviously, $t B_{\mathrm{H} \alpha}$ must be significant in (1) over the Thompson scatter contribution because $r_{\text {patch }}$ differs considerably from $r_{\mathrm{Th}}$.

Inserting the observed total brightness and polarisation ratios into (1) and (2) and eliminating $t B_{\mathrm{Th}}$, we obtain the relation

$\frac{t B_{\mathrm{H} \alpha}}{t B_{\mathrm{Th}^{\prime}}}=\frac{8}{1-18 r_{\mathrm{H} \alpha}}$

Judging from the brightness and polarisation ratios observed, $r_{\mathrm{H} \alpha}$ cannot be higher than $1 / 18$. This low value of the intrinsic $\mathrm{H} \alpha$ polarisation ratio agrees with the low values obtained 
for chromospheric measurements (Gandorfer 2000; Wiehr \& Bianda 2003). Moreover, the ratio $t B_{\mathrm{H} \alpha} / t B_{\mathrm{Th}^{\prime}}$ cannot be lower than 8 . Consequently a large part of the radiation from the core patch (at least $88 \%$ ) must be $\mathrm{H} \alpha$ emission.

Jejčič \& Heinzel (2009) have calculated the ratio $t B_{\mathrm{H} \alpha} / t B_{\mathrm{Th}^{\prime}}$ for various temperatures and densities (note that they assume a different white-light bandwidth $10 \mathrm{~nm}$ instead of $22.5 \mathrm{~nm}$ and a dilution factor $W=0.416$ instead of 0.21 appropriate for $1.5 R_{\odot}$. This causes our white-light intensities to be 1.3 times brighter than the model intensities assumed in their paper. We neglect this factor in view of the approximate nature of our estimate). For the comparatively low densities we expect in the corona, they derive a linear relation, $t B_{\mathrm{H} \alpha} / t B_{\mathrm{Th}^{\prime}}=n_{\mathrm{e}} / 10^{8} \mathrm{~cm}^{-3}$, which only weakly depends on temperature, provided it is below $15000 \mathrm{~K}$. If these conditions hold in our case, the density in the $\mathrm{H} \alpha$ patch would exceed $8 \times 10^{8} \mathrm{~cm}^{-3}$ and would hence be nearly three orders of magnitude of what we estimated for the CME cloud outside of the patch.

The brightness from the core patch in the CME is dominated by $\mathrm{H} \alpha$ radiation. If, reversely, we had assumed $t B_{\text {patch }}$ to be entirely caused by Thomson scatter, we would have obtained a gross overestimate of the density in the CME core. In view of this result, some CME mass estimates in previous studies, where the contribution of the core brightness was not negligible, may have to be revised.

\section{Conclusions}

We showed that the white-light core of the 31 August 2007 CME is clearly identified with the eruptive prominence observed in the EUVI 304 images. To our knowledge, we demonstrate for the first time that this core material is located close to the centre of CME cloud. Moreover, we showed that the major part of the CME core emission, more than $85 \%$ in our case, is $\mathrm{H} \alpha$ radiation and only a small fraction is Thomson-scattered light. We made a rough estimate of the electron density, showing that the density in the $\mathrm{H} \alpha$ patch will exceed by nearly three orders of magnitude what we estimated for the CME cloud outside of the patch.

Acknowledgements. M.M. would like to thank T. Moran and A. Vourlidas for constructive discussions on PR method and electron densities. The authors acknowledge the use of SECCHI data. The contribution of the I.C. and B.I. benefited form support of the German Space Agency DLR and the German ministry of economy and technology under contract 50 OC 0904. B.I. thanks A. Gandorfer for enlightening discussions on the nature of the $\mathrm{H} \alpha$ radiation.

\section{References}

Billings, D. E. 1966, A guide to the Solar Corona (New York: Academic Press) Bommier, V., Sahal-Brechot, S., \& Leroy, J. L. 1986, A\&A, 156, 79 Cremades, H., \& Bothmer, V. 2004, A\&A, 422, 307

Dere, K. P., Wang, D., \& Howard, R. 2005, ApJ, 620, 119 Gandorfer, A. 2000, The Second Solar Spectrum, Vol. I: $4625 \AA$ A to $6995 \AA$ A, ISBN No. 3728127647 (Zurich: VdF)

Heinzel, P., Bommier, V., \& Vial, J. C. 1996, Sol. Phys., 164, 211

Howard, R. A., Moses, J. D., Vourlidas, A., et al. 2008, SSRv, 136, 67

Hyder, L. C., \& Lites, B. W. 1970, Sol. Phys., 14, 147

Jejčič, S., \& Heinzel, P. 2009, Sol. Phys., 254, 89

Kaiser, M. L., Kucera, T. A., Davila, J. M., et al. 2008, SSRv, 136, 5

Leroy, J. L., Bommier, V., \& Sahal-Brechot, S. 1984, A\&A, 131, 33

Lyot, B. 1939, MNRAS, 99, 580

Mierla, M., Inhester, B., Marque, C., et al. 2009, Sol. Phys., 259, 123

Mierla, M., Inhester, B., Rodriguez, et al. 2011, JASTP, in press, doi : $10.1016 /$ j. jastp. 2010.11 .028

Minnaert, M. 1930, ZA, 1, 209

Moran, T. G., \& Davila, J. M. 2004, Science, 305, 66

Morgan, H., \& Habbal, S. R. 2007, A\&A, 471, 47

Poland, A. I., \& Munro, R. H. 1976, ApJ, 209, 927

Sahal-Brechot, S., Bommier, V., \& Leroy, J. L. 1977, A\&A, 59, 223

Tandberg-Hanssen, E. 1995, Science, 269, 111

Thompson, W. T., \& Reginald, N. L. 2008, Sol. Phys., 250, 443

van de Hulst, H. C., Bull. Astron. Inst. Netherland, 11, 135

Vourlidas, A., \& Howard, R. A. 2006, ApJ, 642, 1216

Wiehr, E., \& Bianda, M. 2003, A\&A, 404, 25 